\title{
The Effect of Calcium Carbide Particle Size Distribution on the Kinetics of Hot Metal Desulphurization
}

\author{
J. M. COUDURE and G. A. IRONS
}

Department of Materials Science and Engineering, McMaster University, 1280 Main Street West, Hamilton, Ontario, Canada L8S 4L7.

(Received on August 11, 1993; accepted in final form on October 22, 1993)

\begin{abstract}
The effects of particle size distribution on the kinetics of hot metal desulphurization were investigated by pilot-scale injection. Three different particle size distributions of calcium carbide were injected into $70 \mathrm{~kg}$ heats of carbon-saturated iron. The sulphur contents and oxygen activities were measured during the injection. The reaction in the plume during the injection could be described as a first-order, diffusion-controlled reaction, after an incubation period lasting between 20 and $40 \mathrm{sec}$. This rate constant was found to increase as the particle size decreased. A kinetic analysis based on mass transfer theory was performed considering the total particle size distribution of the calcium carbide. Through this analysis a new average size, directly related to the mass transfer behaviour, was developed. Comparison of the observed and theoretical dependencies of the first-order rate constant on the powder feed rate suggests that the fraction of particles in contact with the melt decreases as the particle size decreases. Finally, issues of scaleup and economic assessment are discussed.
\end{abstract}

KEY WORDS: hot metal; desulphurization; calcium carbide; particles; kinetics.

\section{Introduction}

Desulphurization of iron from the blast furnace is a well-established technology. Many different reagents and processes have been developed; for a comprehensive review of the subject one may refer to Refs. 1) and 2). Among the different reagents used, calcium carbidebased reagents and magnesium-based reagents are currently the most popular.

One important factor in the cost of a particular process is the reagent consumption to reach the aim sulphur content. This factor has become even more important as the sulphur specifications have become more stringent because consumption increases dramatically as the sulphur content decreases. Desulphurization with calcium carbide has been studied in some detail, but the effects of the particle size on reagent consumption and reaction rate have never been systematically investigated. Particle size is an important factor since the interfacial area for reaction is largely determined by the particle size. The major question to be answered is whether the increased cost of grinding and screening to a finer size can be offset by increased reaction rate and efficiency of utilization. This question also suggests that there is an optimum size "for particular conditions of reagent composition, aim sulphur content, and grinding cost functions. The objective of the present work is to provide some understanding of the particle size-reaction rate relationships for calcium carbide reagents to guide both steelmakers and reagent producers.

\section{Fundamental Aspects}

Figure 1 summarizes the current level of understanding of hot metal desulphurization by calcium carbide injection. ${ }^{3)}$ Powder is injected, along with a carrier gas, into molten metal through a lance; a jet is created at the outlet of the lance that penetrates into the melt until its momentum is dissipated. Then, a three-phase plume is created which rises in the melt, and finally the particles of calcium carbide, reacted or unreacted, are incorporated into the top slag. The reaction between calcium carbide and the sulphur dissolved in the hot metal:

$$
\mathrm{CaC}_{2(\mathrm{~s})}+\underline{\mathrm{S}}=\mathrm{CaS}_{(\mathrm{s})}+2 \mathrm{C}_{(\mathrm{s})}
$$

takes place mainly in the rising plume (the so-called transitory reactor) where the interfacial area between the particles and liquid iron is very high. The reaction goes on, to a lesser extent, at the slag-metal interface, (the permanent reactor).

The mechanism of desulphurization with calcium carbide was first studied by Talballa et $a l .{ }^{4)}$ According to their results, calcium carbide, which is solid at the temperature of liquid iron $\left(1350^{\circ} \mathrm{C}\right)$, partially dissociates into calcium vapour and a layer of graphite. This calcium vapour then reacts with sulphur dissolved in iron to form a layer of calcium sulphide along with the graphite layer. The layers of graphite and calcium sulphide progressively thicken to form a barrier to calcium vapour diffusion vapour which slows the rate. Based on this mechanism, 


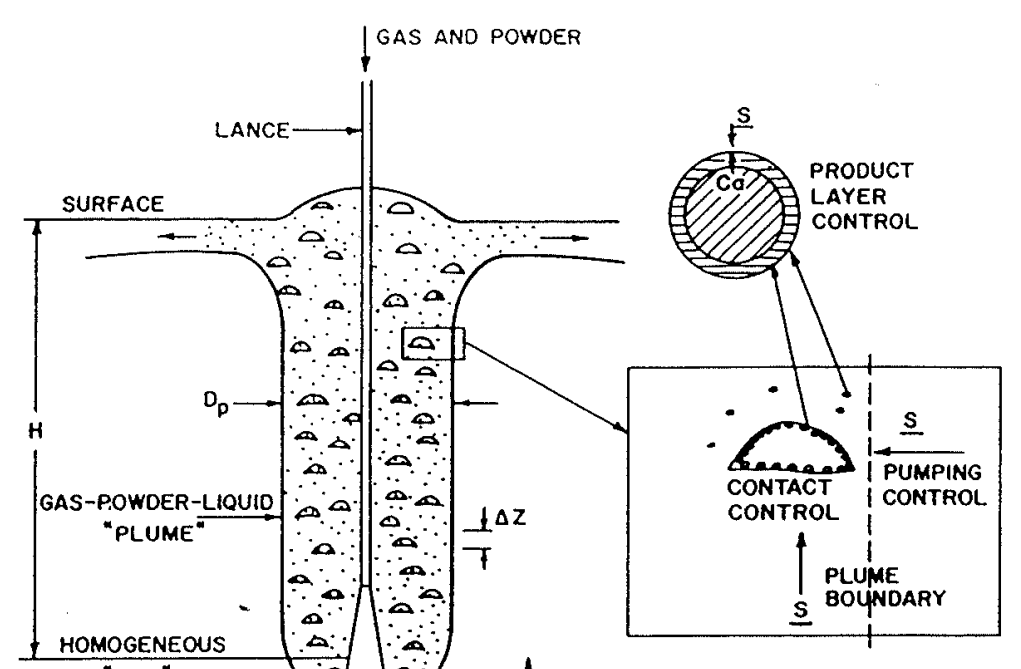

Fig. 1.

Schematic representation of the physical and chemical phenomena in the rising plume. The carrier gas and injected particles rise and react through the plume. The inset indicates that particles may be on the bubble interface or in the liquid. At individual particles, calcium vapour diffuses through the product layers.

\section{lz}

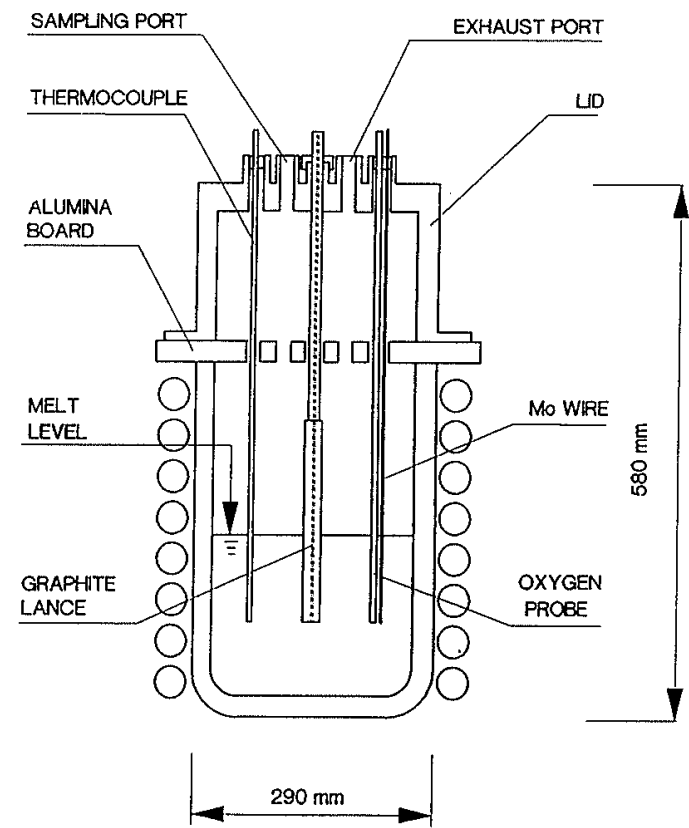

Fig. 2. Schematic diagram of the crucible and lid assembly, to scale.

Chiang et $a l .^{3)}$ developed a model for calcium carbide desulphurization in the plume. They showed that the reaction was controlled by the diffusion of sulphur through the particle boundary layer, rather than the diffusion of calcium vapour in the product layer. Their experimental results confirmed that the reaction could be described as a first-order, diffusion-controlled reaction. In further work, Irons ${ }^{5)}$ examined mixing as a possible controlling step in the desulphurization process. He concluded that mixing was always fast enough not to be rate controlling. Consequently, the reaction is controlled by diffusion through the boundary layers around the particles.

To calculate this diffusion-controlled rate, one must know whether the particles are contacting the melt or remaining on the carrier gas bubbles. This is an important point because calcium carbide is not wetted by liquid iron. The particle-liquid contact pattern during calcium carbide powder injection was first assessed by Chiang et $a l .{ }^{3)}$ They performed experiments on $2.5 \mathrm{t}$ of hot metal and investigated the dependency of the desulphurization rate constant on powder feed rate and gas flow rate. By comparison of the experimental results against a model based on transport phenomena, they concluded that only 20 to $40 \%$ of the particles contacted the melt effectively during calcium carbide injection. The remaining particles were on the carrier gas bubbles interfaces. A similar degree of contact has been observed by Zhao in experiments conducted with the present apparatus. ${ }^{6)}$

The oxygen activity has been found to greatly affect process efficiency. Zhao found that desulphurization and deoxidation occurred simultaneously during calcium carbide powder injection by Reactions (1) and (2):

$$
\mathrm{CaC}_{2(\mathrm{~s})}+\underline{\mathrm{O}} \rightleftarrows \mathrm{CaO}_{(\mathrm{s})}+2 \mathrm{C}_{(\mathrm{s})}
$$

Furthermore, his results showed that the oxygen activity before the injection was controlled by the silicon content in the iron (i.e., the silicon/silica equilibrium), and that the rate of desulphurization was increased by low oxygen activity in the melt. ${ }^{6}$ )

\section{Experimental Apparatus and Procedure}

\subsection{Apparatus}

Seventy kilograms of iron were melted in magnesia crucibles contained in a $75-\mathrm{kW}$ induction furnace. The crucibles were $230 \mathrm{~mm}$ inside diameter and $365 \mathrm{~mm}$ high. A specially-designed steel lid covered the melt to provide protection from the atmosphere. The lid was fitted with ports for sampling, temperature and oxygen activity measurements as shown in Fig. 2. Graphite lances (3 mm i.d., $25 \mathrm{~mm}$ o.d.) were immersed $100 \mathrm{~mm}$ into the melt. These lances were connected to a pressurized screw-type powder dispenser from which the calcium carbide was injected. This powder dispenser allowed the nitrogen flow rate and powder feed rate to be controlled independently. The gas flow rate was controlled by a needle valve, whereas the powder feed rate was adjusted by controlling the rotation rate of the screw.

The weight losses of the powder dispenser were measured by a load cell, and the gas flow rate by a gas flow rate transducer. Melt temperatures were monitored 
by a $\mathrm{Pt} / \mathrm{Pt}-\mathrm{Rh} 13 \%$ thermocouple immersed into the melt. The oxygen activity in the hot metal was measured before and after each injection, using commercially available disposable, single-use oxygen probes supplied by Electro-Nite (Model CELOX 2/CLL). The signals from the loadcell, the gas flow rate transducer, the thermocouple and the continuous oxygen probe were collected in a data-logging microcomputer.

\subsection{Procedure}

After the iron was melted, the lid was placed on the top of the crucible. The bath temperature was stabilized to $1350 \pm 10^{\circ} \mathrm{C}$. Then, $\mathrm{FeS}_{2}$ and ferrosilicon were plunged into the melt to adjust the starting chemical composition of the iron (between 0.05 and $0.07 \% \mathrm{~S}$ and $0.7 \% \mathrm{Si}$ ). One spoon sample was taken. Then, temperature and oxygen activity were measured with a disposable, single-use probe. The lance was lowered into the melt. Sometimes, a continuous oxygen probe was dipped into the melt. After about $2 \mathrm{~min}$ of nitrogen bubbling necessary to set up all the experimental devices, the gas flow rate was adjusted and the power to the induction furnace shut off. The powder was injected for 2 to $4 \mathrm{~min}$. During the injection, as many pin samples as possible were taken by suction. After the powder injection was stopped, nitrogen bubbling was continued for about 2 or $3 \mathrm{~min}$ and pin samples were also taken. The lid was then removed from the crucible and the resulting top slag skimmed; the melt was prepared for another injection by adjusting the sulphur content. Typically four injections were made during one heat.

\subsection{Chemical Analyses}

Pin samples taken during the injections were crushed and then analyzed for sulphur and carbon contents using a combustion and infra red absorption apparatus (Leco, Model CS-444). The spoon samples were analyzed by optical emission spectrometry (Jarrell-Ash Inductively Coupled Argon Plasma Solid Sampling System 9000) for total silicon, manganese and aluminum contents.

The nominal chemical composition of the iron used in this study (supplied by QIT Fer et Titane, Sorel, Québec, Canada) was $4.2 \% \mathrm{C}, 0.02 \% \mathrm{Mn},<0.001 \% \mathrm{Al}, 0.1 \%$ $\mathrm{Si}$.

\section{Characterization of the Calcium Carbide Powders}

In the present study, the three different particle size distributions of calcium carbide will be called types A, $\mathrm{B}$ and $\mathrm{C}$. They were obtained by screening commercially available technical calcium carbide, produced and supplied by Pechiney Electrométallurgie, France. The technical calcium carbide contained nominally $77.5 \%$ $\mathrm{CaC}_{2}, 2.4 \%$ free $\mathrm{C}$ and the balance $\mathrm{CaO}$. The screening operations were conducted under inert atmosphere to reduce reactions of calcium carbide with air, using a Rotap-dry screening type apparatus. Several samples were taken at random for chemical analysis and particle size determinations.

\subsection{Chemical Analyses}

Two samples were analyzed for each type of powder;
Table 1. Composition of calcium carbide powders

\begin{tabular}{cccccc}
\hline Powder & $\% \mathrm{CaC}_{2}$ & $\% \mathrm{Ca}(\mathrm{OH})_{2}$ & $\% \mathrm{CaCO}_{3}$ & $\% \mathrm{C}_{\text {free }}$ & $\% \mathrm{CaO}^{*}$ \\
\hline A & 67.3 & 9.3 & 0 & 3.2 & 20.2 \\
B & 78.2 & 3.3 & 0 & 2.4 & 16.1 \\
C & 79.3 & 2.2 & 0 & 2.4 & 16.0 \\
\hline
\end{tabular}

* \% CaO determined by difference.

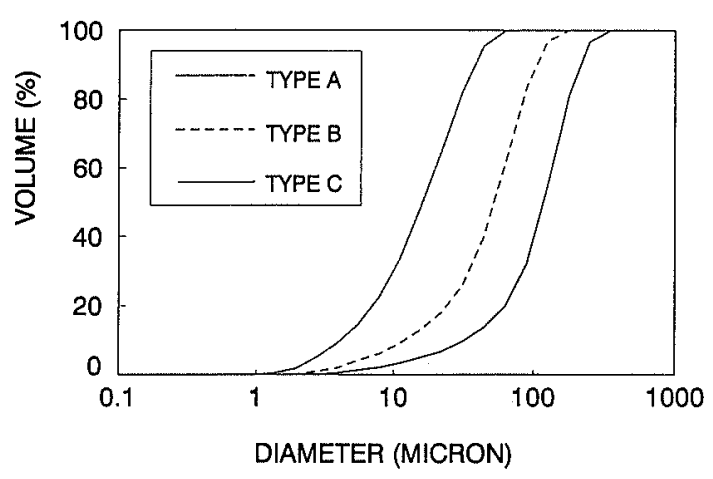

Fig. 3. Cumulative volume distributions for the powders.

the analyses were performed by wet chemistry, including calcium carbide content (by measuring the amount of $\mathrm{C}_{2} \mathrm{H}_{2}$ evolved), calcium carbonate, free carbon and moisture (calcium hydroxide) contents. Table 1 summarizes the results.

Commercial calcium carbide always contained unreacted lime from the manufacturing process. As mentioned above, this Pechiney material nominally contains $78 \%$ calcium carbide; however, Type A powder contains less than $70 \%$ calcium carbide, and a higher content of calcium hydroxide. This material was the finest material tested which made it more sensitive to reaction with atmospheric moisture.

\subsection{Particle Size Determination}

Particle size measurements were performed using a Microtrac II Particle size Analyzer (Leeds \& Northup Instruments, St Petersburg, Florida) which operates over particle sizes ranging from 0.1 to $1000 \mu \mathrm{m}$, based on a light diffraction and scattering principles. ${ }^{7,8)}$ The particles must be completely dispersed in a liquid to ensure that one is not measuring the size distribution of particle agglomerates. For calcium carbide powders, Isopropyl Alcohol (IPA) was found to be very effective in improving the dispersability of the particles and reduce inter-particle attractions. ${ }^{9)}$ Figure 3 shows the cumulative volume distributions for the three types of powders.

It is convenient to use a characteristic dimension of the powder determined from its particle size distribution, such as an average particle size. However, several types of averaging procedures can be employed: ${ }^{10)}$ The median of the volume distribution, $d_{\text {vol }}^{m}$, can be read directly from the cumulative volumetric distribution (Figure 3). The mean size corresponds to the arithmetic average of the distribution and is given by:

$$
\bar{d}_{\mathrm{vol}}=\frac{\sum_{i}\left[d_{p i} \% \mathrm{Vol}_{i}\right]}{\sum_{i} \% \mathrm{Vol}_{i}}
$$


Table 2. Average sizes for the calcium carbide powders.

\begin{tabular}{cccccccc}
\hline \multirow{2}{*}{ Powder } & \multicolumn{2}{c}{ Median sizes } & & \multicolumn{3}{c}{ Mean sizes } \\
\cline { 2 - 3 } \cline { 5 - 7 } & $d_{\text {vol }}^{m}$ & $d_{\text {area }}^{m}$ & & $d_{\text {vol }}$ & $d_{\text {arca }}$ & $d_{k a}$ \\
\hline A & 16.7 & 5.90 & & 20.5 & 9.70 & 6.03 \\
B & 51.8 & 13.1 & & 55.3 & 24.9 & 11.8 \\
$\mathrm{C}$ & 113. & 21.1 & & 116. & 49.5 & 20.9 \\
\hline
\end{tabular}

where $(\% \mathrm{Vol})_{i}$ is the percentage of the volume in channel $i$ of the distribution, and $d_{p i}$ is the average diameter in that channel.

Median and mean diameters can be calculated with respect to the surface areas of the particles. The volume percent for each channel can be converted to an area percent:

$$
\% \text { Area }_{i}=\frac{\% \mathrm{Vol}_{i} / d_{i}}{\sum_{i} \% \mathrm{Vol}_{i} / d_{i}}
$$

The median area diameter can be read directly from a cumulative area average distribution. The mean area diameter can be calculated from:

$$
\bar{d}_{\mathrm{area}}=\frac{\sum_{i} d_{p i} \% \mathrm{Area}_{i}}{\sum_{i} \% \mathrm{Area}_{i}}
$$

Table 2 contains the mean and median volume and area diameters.

One can see that there are considerable differences between the various characteristic dimensions of the particles. The mean and median sizes calculated from the same distribution of a powder are different because the distributions are not statistically normal. Areaaveraging places more weight on the smaller diameters because of the high surface-to-volume ratio of these particles. The choice of the proper averaging procedure will be addressed in the Discussion.

\section{Experimental Results}

\subsection{Sulphur Content}

The sulphur contents of the iron during typical experiments are shown in Fig. 4. For an initial period the sulphur content remains constant; this will be called the incubation time. After that, the sulphur contents decline rapidly. The incubation period has been observed in several other experiments. ${ }^{3,6)}$ It occurs because there was no top slag in these experiments; consequently, the calcium sulphide reaction product could not be absorbed at the top of the melt, causing the sulphur to revert to the melt. Once a layer of unreacted and reacted particles accumulate on the surface, this mixture will start to desulphurize as well. It should be noted that the "top slag" is not a slag in the conventional sense (a solution of oxides), but will be referred to as such for convenience.

For the two injections in Fig. 4, the gas and particle injection rates were very similar, thus, the major difference is the particle size. Clearly the finer particles de-

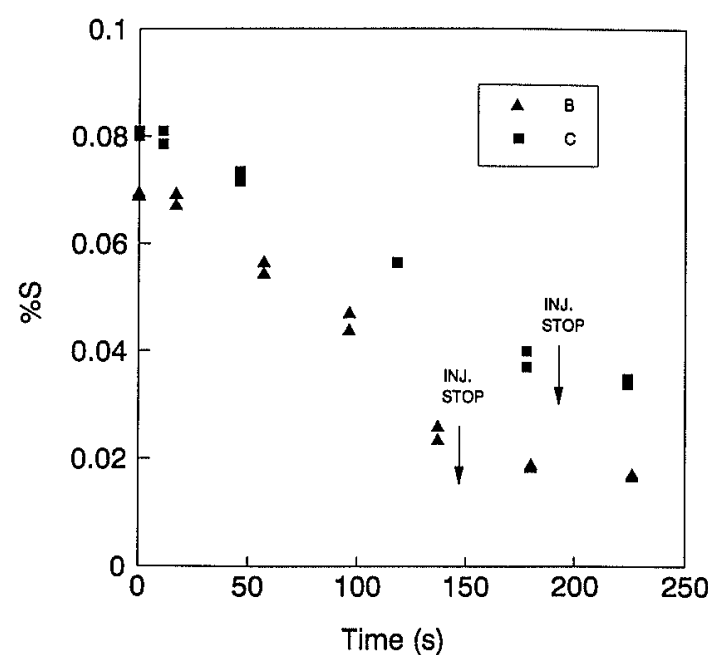

Fig. 4. Typical sulphur vs. time curves showing the effect of the particle size for two injections performed under similar conditions of powder feed rate $(200 \pm 10 \mathrm{~g} / \mathrm{min})$ and gas flow rate (13 1 SLPM), but different particle size distributions.

sulphurize more quickly.

After powder injection, gas bubbling was continued at approximately the same rate as during injection to examine the reaction rate with the top slag. One can see that the rate of desulphurization is slower with only the top slag.

\subsection{Oxygen Activity}

In previous work on hot metal desulphurization with calcium carbide, it was found that the oxygen activity greatly affected desulphurization rates; the rate increased as the oxygen activity decreased. ${ }^{6,11)}$ It was also found that the oxygen activity before powder injection was controlled by the silicon/silica equilibrium or the carbon/carbon monoxide equilibrium, whichever was lower. Therefore, in the design of the present experiments particular attention was placed on the control of the oxygen activity in hot metal. The silicon content in the hot metal was adjusted to $0.7 \% \mathrm{Si}$ before each injection; the carbon content remained at $4.2 \%$ without any adjustment.

The oxygen activity was first measured using disposable, single-use oxygen probes from Electro-Nite, before and after each injection. The measured oxygen activities were always slightly higher than those calculated from the silicon/silica equilibrium, which was consistent with previous work. ${ }^{611)}$ On average, the starting oxygen activity was $2.8(\mathrm{ppm})$ and the final oxygen activity 2.1 (ppm).

\section{Interpretation of Experimental Results}

\subsection{Plume Desulphurization Rate}

It has been shown in previous work that the rate of desulphurization can be described as a first-order, diffusion-controlled reaction, after the incubation period $^{3,6)}$;

$$
\frac{d \% \mathrm{~S}}{d t}=-K\left(\% \mathrm{~S}-\% \mathrm{~S}^{*}\right)
$$




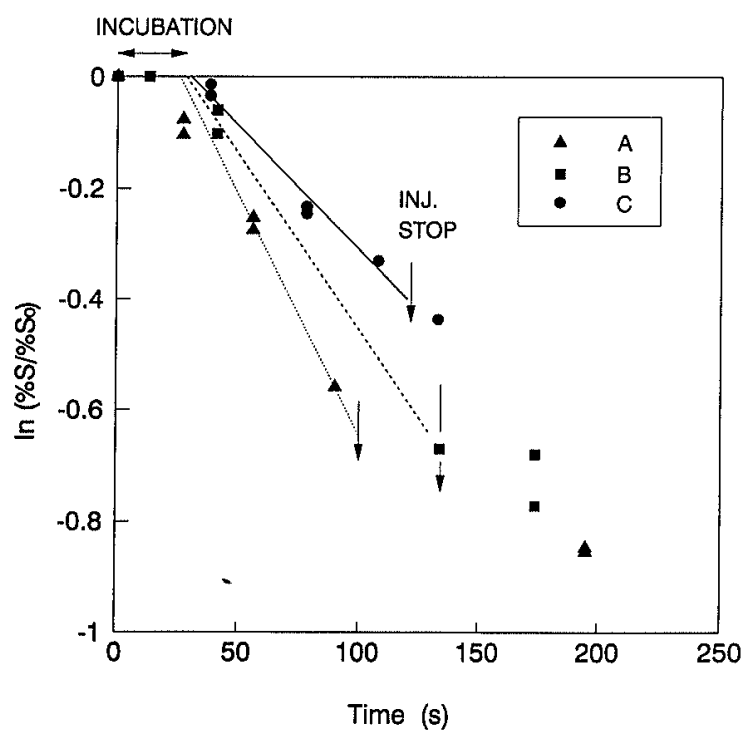

Fig. 5. The logarithm of the fraction of remaining sulphur $v s$. time demonstrating the effect of the particle size for a set of similar injection conditions $(475 \pm 35 \mathrm{~g} / \mathrm{min}$ powder feed rate and $9 \pm 1$ SLPM gas flow rate).

where $K$ is the volumetric rate constant for the reaction.

In the case of calcium carbide, the equilibrium sulphur content is very small compared to $\% \mathrm{~S}$, thus $\% \mathrm{~S}^{*}$ can be neglected in Eq. (6). Rearranging and integrating yields:

$$
\ln \frac{\% \mathrm{~S}}{\% \mathrm{~S}_{0}}=-K t
$$

where $\% \mathrm{~S}_{0}$ is the initial sulphur content in the melt. If the logarithm of the fraction of remaining sulphur is plotted against time, the slope will be $K$. These curves are plotted for three typical injections in Fig. 5. One may see that the curves are linear after the incubation period. The slopes of the lines, which were obtained from linear regression analysis, represent the first-order rate constants. The rate of desulphurization, after the incubation period, increases as the particle size decreases.

To determine how much desulphurization occurred by reactions with the top slag, gas injection was continued after the powder dispenser was shut off. It is reasonable to assume that agitation conditions at the top slag interface are mainly influenced by the gas injection rate, rather than by the powder injection rate. Therefore, the rate of desulphurization immediately after the dispenser was shut off reflects the contribution of the top slag conditions to the total desulphurization rate during injection. Data from the holding periods, such as Fig. 4, when plotted to determine the first order rate constants, were also linear. There was no dependence of the rate constants in the holding period on particle size.

To determine the effect of the particle size on the desulphurization rate during particle rise in the melt, the rate of desulphurization after powder injection was subtracted from the measured rate during injection. The resulting first-order rate constants are plotted as a function of the powder injection rate in Fig. 6. Clearly the first-order rate constant increases with the solids rate.

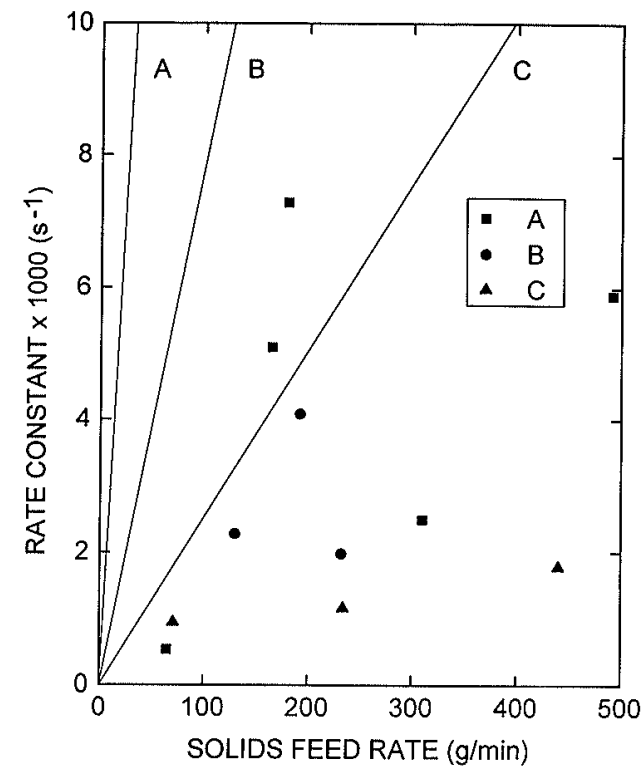

Fig. 6. Dependency of the first-order desulphurization rate constant on the powder feed rate for the various calcium carbide particle size distributions. The lines represent relationships developed from the mass transfer theory to the solid particles of calcium carbide, assuming that all the particles contact the melt.

\subsection{Fundamental Interpretation of Desulphurization Rate}

The objective of this section is to interpret the rate data with mass transfer theory. As discussed earlier, due to the non-wettability of calcium carbide, the particles may contact liquid iron or may remain positioned at the gas-liquid interface.

The rate constant $K$ for desulphurization in the plume, defined by Eq. (6), can be evaluated assuming all the particles enter the melt, based on previous modelling., ${ }^{3,6)}$ $K$ can be expressed as:

$$
K=\frac{k_{p} A_{p}}{V}=\left(k_{p} a\right)_{\mathrm{plume}}
$$

where $a$ is the total area of particles in contact with the melt per unit volume of melt.

The mass transfer coefficient for particles in the melt, $k_{p}$, can be estimated from the Ranz-Marshall correlation for solid spheres ${ }^{12)}$ :

$$
S h=\frac{k_{p} d_{p}}{D_{s}}=2+0.6 R e^{1 / 2} S c^{1 / 3}
$$

The slip velocity of micron-size particles is very small, resulting in Reynolds Numbers much less than unity. Consequently, the Sherwood Number approaches the limiting value of 2, for radial diffusion. A similar conclusion can be reached by using Yano's ${ }^{13}$ correlation for mass-transfer to particles in gas-agitated vessels:

$$
S h=2+0.4\left(\frac{\varepsilon d_{p}^{4}}{v^{3}}\right)^{1 / 4} S c^{1 / 3}
$$

However, it is not readily apparent which diameter should be used in Eqs. (8) to (10) to calculate the mass transfer coefficient. Table II shows that average particle diameters can vary tremendously depending on the 
averaging procedure used. The appropriate averaging procedure can be determined by applying mass transfer principles to each channel of the volume particle size distribution, and then summing the effects over all channels.

For each channel of the distribution, a Sherwood Number of 2 can be used, thus:

$$
k_{p i}=\frac{2 D_{s}}{d_{p i}}
$$

where $k_{p i}$ is the mass transfer coefficient for particles in channel $i$ of the volume distribution and $d_{p i}$ is the mean size in that channel.

Summing over.all channels yields the total volumetric mass transfer coefficient in the plume:

$$
\left(k_{p} a\right)_{\mathrm{plume}}=\sum_{i} k_{p i} a_{i}
$$

If all of the particles injected contact the melt, then the total volume of particles per unit volume of melt is given by:

$$
V_{\mathrm{CaC}_{2}}=\frac{W_{p} t_{r}}{\rho_{p}} \frac{1}{V}
$$

From this expression, one can calculate the area of the particles per unit volume of melt corresponding to the particles in channel $i$ :

$$
a_{i}=\frac{6}{d_{p i}} \frac{\% \mathrm{Vol}_{i}}{100} \frac{W_{p} t_{r}}{\rho_{p} V}
$$

where the residence time of the particles in the melt is:

$$
t_{r}=\frac{L_{p}}{u_{p}}
$$

If one assumes that the rising distance of the particles is equal to the lance immersion, Eqs. (11) to (13) can be rearranged to yield:

$$
k_{p i} a_{i}=\frac{12 W_{p} D_{s} L_{p}}{\rho_{p} u_{p} V} \frac{\% \mathrm{Vol}_{i}}{100} \frac{1}{d_{p i}^{2}}
$$

Finally, the rate constant $K$ for all the particles in the melt can be obtained by summing Eq. (16) over all channels:

$$
K=\left(k_{p} a\right)_{\text {plume }}=\frac{12}{100} \frac{W_{p} D_{s} L_{p}}{\rho_{p} u_{p} V} \sum_{i} \frac{\% \mathrm{Vol}_{i}}{d_{p i}^{2}}
$$

If one wants to write the total mass transfer coefficient in terms of the appropriate mean diameter for mass transfer, $\bar{d}_{k a}$ :

$$
k_{p}=\frac{2 D_{s}}{\bar{d}_{k a}}
$$

then Eq. (17) can be rewritten as:

$$
K=\frac{12 W_{p} D_{s} L_{p}}{\rho_{p} u_{p} V \bar{d}_{k a}^{2}}
$$

Comparing Eqs. (17) and (19), $\bar{d}_{k a}$ is:

$$
\bar{d}_{k a}=\left(\frac{\sum_{i} \% \mathrm{Vol}_{i}}{100 d_{p i}^{2}}\right)^{-2}
$$

which can be interpreted as the equivalent diameter, for a diffusion-controlled process, to yield the observed rate of mass transfer when all the particles are reacting. Table 2 shows the values of $\bar{d}_{k a}$, calculated according to Eq. (20), for the three types of powders.

Equation (19) predicts that $K$ is a linear function of the powder feed rate. In Fig. 6, $K$ is plotted as a function of the powder feed rate for the three powders to be compared with the experimental results. (For these calculations, the lance immersion was $0.1 \mathrm{~m}$, the particle rising velocity was estimated to be $0.12 \mathrm{~m} / \mathrm{s}$ from previous work on plumes, ${ }^{14)}$ the sulphur diffusivity was taken as $1.5 \times 10^{-9} \mathrm{~m}^{2} / \mathrm{s}^{15)}$ and the calcium carbide bulk density was taken as $2200 \mathrm{~kg} / \mathrm{m}^{16)}$ ). One may see that the estimated rate constant is always higher than the ones experimentally observed. While there is some scatter in the data, it appears that the rate constants do not continue to increase with the injection rate. In the development of Eq. (19) it was assumed that all of the particles entered the melt. However, this is probably not true as calcium carbide is not wetted by liquid iron. Thus, only a fraction of the calcium carbide particles contacts the melt, the remaining particles being positioned at the bubbles interfaces or even suspended in the bubbles. Previous work on calcium carbide desulphurization has similarly shown that only a fraction of the particles are in contact with the melt and that this fraction decreases as the injection rate increases. ${ }^{6,11}$ )

\section{Discussion}

Theoretically, Eq. (16) shows that the first order rate constant should be inversely proportional to the square of the particle diameter. For a particle size distribution, Eq. (19) shows that the rate constant is similarly inversely proportional to the square of $\bar{d}_{k a}$. This would imply that dramatic improvements in reaction rate and reagent utilization can be obtained from finer particles. Unfortunately, such large improvements were not observed in the present experiments. Figure 6 shows that the observed rates are considerably below the theoretical limit which is attributed to incomplete particles with the melt. Figure 7 shows the data re-plotted as the ratio of the actual to theoretical rates. It can be seen that the smallest particles have the lowest ratio which is probably due to the poorest particle-liquid contact.

In Fig. 8 the observed rate constants are plotted as a function of the average particle size. It may be seen that there is a strong dependence on the particle size; logarithmic regression yields the following relationship:

$$
K=10^{(-9.05 \pm 1.97)} \bar{d}_{k a}{ }^{-(1.31 \pm 0.28)} \quad\left(r^{2}=0.84\right)
$$

The dependence on particle size $(-1.31)$ is weaker than expected if all the particles enter the melt $(-2)$; however, it should be noted that this is still a strong dependency.

The present experiments were conducted on a much smaller scale than industrial operations. Superficial gas 


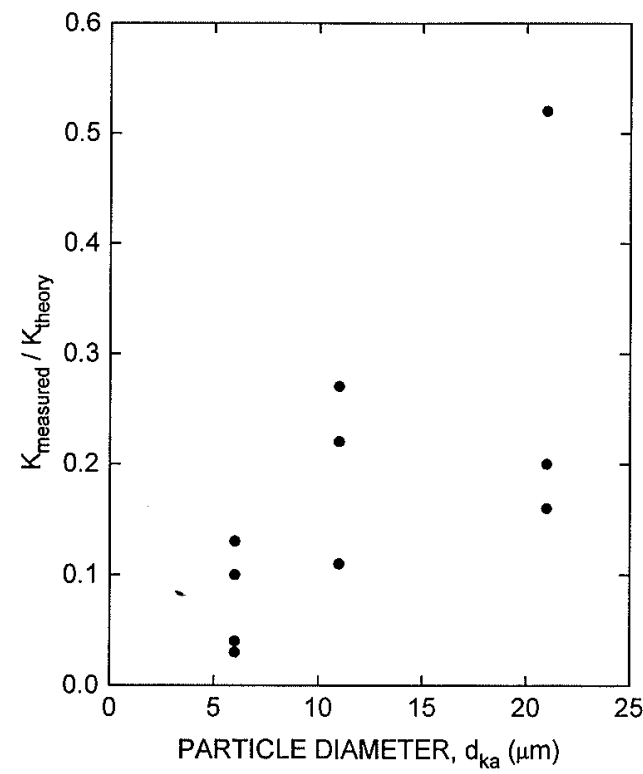

Fig. 7. The ratio of the measured first order rate constant to that calculated assuming that all the particles contact the melt. This ratio is considerably lower for the finer particles.

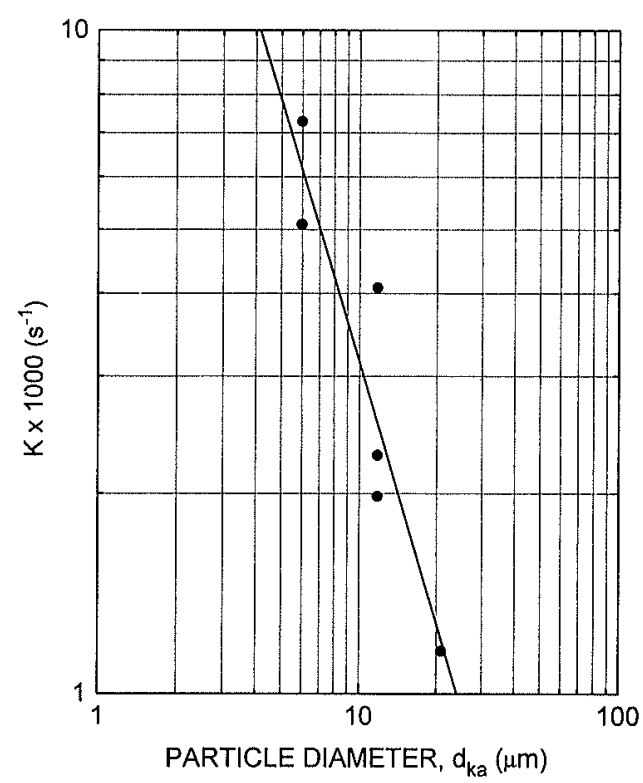

Fig. 8. The measured first-order rate constant plotted as a function of the particle diameter calculated from transport theory, $d_{k a}$, in the solids injection rate range of $190 \pm 50 \mathrm{~g} / \mathrm{min}$. The regression line, Eq. (21), is also plotted.

velocities were in the range 10 to $30 \mathrm{~m} / \mathrm{s}$, corresponding to a Mach Numbers of 0.03 to 0.09 , with solid loadings in the range 5 to $60 \mathrm{~kg}$ of solids $/ \mathrm{Nm}^{3}$ of carrier gas. Commercial operations would use larger diameter lances, but have gas velocities in the 30 to $100 \mathrm{~m} / \mathrm{s}$ and solid loadings 60 to $200 \mathrm{~kg} / \mathrm{Nm}^{3}$. There has been a considerable amount of work carried out on the regimes of flow and contacting patterns with the liquid. ${ }^{17)}$ Commercial operations with fine particles, such as calcium carbide, would be expected to operate in the jetting regime, as shown in Fig. 1. In such cases, the gas and particles flow with virtually the same velocity, penetrating into the melt until their downward momentum is dissipated. For the present experiments, calculations suggest that the flow is not fully jetting because the calculated jet length is shorter than a bubble diameter.

In the fully jetting case, the particles have little opportunity to contact the liquid until they reach the bottom of the jet. In the present experiments, the particles would contact the bottom surface of the bubble, close to the lance. Therefore, the conditions for particle-liquid contact in the present experiments are similar to those at the bottom of the jet in the full-scale operations. It is surmised that the particles position themselves as dictated by the surface tension forces, similarly to mineral flotation. Since calcium carbide is not wetted by liquid iron, this would account for the previous observations that only 30 to $50 \%$ of the particles contact the melt. ${ }^{3,6)}$ In the present work, the ratio is even lower for fine particles (Fig. 7) which may be due to the higher surface area-to-volume ratio of the particles, making them even more dominated by surface tension forces. Higher up in the plume, the bubbles are simply rising due to buoyancy and there is little reason to expect the number of particles on the bubbles to change dramatically. Therefore, similar dependencies on particle size can be expected in full-scale operations.

One aspect that complicated the present analysis was the effect of the top slag. Initially, there was no top slag present, and it took some time (the incubation time) for the accumulating slag to become effective in absorbing sulphur. For desulphurization of hot metal from the blast furnace, the effect of the top slag must also be considered. Sometimes there may be an incubation period. ${ }^{3)}$ In any case, the refining rate of the top slag must be subtracted from the total refining rate, as was done in the present experiments to determine the effects of particle size distribution on the rate. Consequently, the effect of particle size on the total refining rate will not be as strong as the effect on the plume reaction rate.

The most straightforward way to carry out an economic analysis of the benefits of decreasing particle size is to examine incremental improvements in reagent utilization against the incremental cost of grinding and screening. The first order rate constant for the plume reactions can be related to the utilization by combining Eq. (7) with a sulphur balance for the particle and melt:

$$
U=\frac{\% \mathrm{~S}_{0}[1-\exp (-K t)] W_{\mathrm{Fe}}}{\alpha W_{R}}
$$

where $\alpha$ is the mass of sulphur removed per unit mass of reagent based on Eq. (1), and $W_{\mathrm{Fe}}$ and $W_{\mathrm{R}}$ are the weights of iron and reagent, respectively. (This relationship ignores the effect of the top slag). This nonlinear relationship proves that there are decreasing returns in increasing the rate constant as the stoichiometric utilization approaches unity. (For very low rate constants, the relationship becomes linear because the quantity in the square brackets in Eq. (22) approaches $K t$ ). Thus, the possible improvements for each plant depend on the current utilization, starting sulphur contents, extent of top slag reactions and the incremental cost of grinding. 
Another aspect to be considered from the industrial point of view is the flowability of the powders. Indeed, it is very important to impart consistent flow behaviour of the reagents and it is expected that decreasing the particle size would decrease the powder flowability by agglomeration ${ }^{9)}$. No quantitative measurement of the flowability of the powders used in this study have been undertaken (mainly because there is no universally accepted precedure ${ }^{18)}$ ). However, the tendency towards agglomeration could be observed by eye, especially for powder A. Furthermore, a smaller fraction of powder A was found to react (Fig. 7). It has been found that surface treatments of the reagents improve the flowability of the particles and the consistency of the process. Such flow aids improve the flowability of the powders by reducing the inter-particle attraction. The flow aids are usually organic polar liquids that are inert with respect to the desulphurizing reagent, such as isopropyl alcohol. ${ }^{19,20)}$

\section{Conclusions}

Pilot-scale experiments were performed to investigate the effect of the particle size of calcium carbide powder on the desulphurization process. The main conclusions of this study are the following:

(1) The reaction between $\mathrm{CaC}_{2}$ particles and sulphur dissolved in hot metal during powder injection could be described as a first-order, diffusion-controlled reaction, after an incubation period lasting between 20 and $40 \mathrm{sec}$.

(2) Decreasing the particle size of the calcium carbide increased the desulphurization rate, which has not been previously shown.

(3) A kinetic analysis in terms of mass transfer to solid calcium carbide particles considering the total particle size distribution has lead to the determination of an appropriate average size to evaluate the efficiency of the process.

(4) The model suggested that a significant fraction of the particles do not contact the melt and remain positioned on the carrier gas bubbles; furthermore, the extent of particle-liquid contact decreases with decreasing particle size.

\section{Acknowledgments}

The authors would like to thank Mr. O. Kelly for his assistance with the experimental work, Mr. M. Van Oosten with the chemical analysis, and Mr. I. A. Cameron, formerly with Cyanamid Canada Inc., for performing particle size analysis. The financial support and encouragement from the Pechiney Electrométallurgie Research Centre (LEC, Chedde, France), particularly from Dr. G. Nussbaum, are gratefully acknowledged. The donations of iron by QIT Fer et Titane Inc., oxygen probes by Electro-Nite and calcium carbide powders by Pechiney Electrométallurgie, France are sincerely appreciated.

\section{Nomenclature}

$a:$ surface area of particles per volume of melt $\left(\mathrm{m}^{-1}\right)$

$A$ : surface area of the melt $\left(\mathrm{m}^{2}\right)$
$A_{p}: \quad$ surface area of particles in the melt $\left(\mathrm{m}^{2}\right)$

$d_{\text {area }}^{m}:$ median diameter of the area distribution (m)

$\bar{d}_{\text {area }}:$ mean diameter of the area distribution (m)

$\bar{d}_{k a}:$ mean diameter of the distribution for mass transfer $(\mathrm{m})$

$d_{p}:$ particle diameter $(\mathrm{m})$

$d_{i}$ : mean diameter in channel $i$ of the volume distribution $(\mathrm{m})$

$d_{\mathrm{vol}}^{m}:$ median diameter of the volume distribution (m)

$\bar{d}_{\mathrm{vol}}:$ mean diameter of the volume distribution (m)

$D_{s}$ : diffusivity of sulphur in iron $\left(\mathrm{m}^{2} / \mathrm{s}\right)$

$k_{p}$ : mass transfer coefficient $(\mathrm{m} / \mathrm{s})$

$k_{p i}$ : mass transfer coefficient for particles in channel $i(\mathrm{~m} / \mathrm{s})$

$K$ : first-order rate constant for desulphurization $(1 / \mathrm{s})$

$L_{p}: \quad$ rising distance for particles in the melt (m)

Re: particle Reynolds number

$\% \mathrm{~S}_{0}$ : initial sulphur content in the melt

$\% \mathrm{~S}^{*}$ : sulphur content at equilibrium

Sc: Schmidt number

$S h$ : Sherwood number

$t:$ time (s)

$t_{r}:$ residence time of the particles in the melt (s)

$u_{p}:$ particle velocity in the plume $(\mathrm{m} / \mathrm{s})$

$U:$ utilization of reagent

$V:$ volume of melt $\left(\mathrm{m}^{3}\right)$

$\% \mathrm{Vol}_{i}$ : volume percent of particles in channel $i$ of the volume distribution

$\%$ Area $_{i}$ : area percent of particles in channel $i$ of the area distribution

$W_{\mathrm{Fe}}$ : mass of iron $(\mathrm{kg})$

$W_{p}:$ powder feed rate $(\mathrm{kg} / \mathrm{s}$ or $\mathrm{g} / \mathrm{min})$

$W_{R}$ : mass of reagent added $(\mathrm{kg})$

$\alpha$ : stoichiometric mass of sulphur removed per mass of reagent

$\varepsilon$ : energy dissipation, defined as bubble slip velocity times gravitational acceleration $\left(\mathrm{m}^{2} / \mathrm{s}^{3}\right)$

$\rho_{p}:$ particle density $\left(\mathrm{kg} / \mathrm{m}^{3}\right)$

$\rho_{l}:$ liquid density $\left(\mathrm{kg} / \mathrm{m}^{3}\right)$

$v$ : kinematic viscosity of the melt $\left(\mathrm{m}^{2} / \mathrm{s}\right)$

\section{REFERENCES}

1) P. J. Koros: Proc. of SCANINJECT III, Mefos and Jernkontoret, Lulea, Sweden, (1983), 24.

2) I. A. Cameron: Chap. 17, Blast Furnace Ironmaking, Lecture Notes, McMaster Univ., Hamilton, ON, (1992), 17-1.

3) L. K. Chiarg, W. K. Lu, G. A. Irons and I. A. Cameron: Trans. ISS, (1990), 35.

4) M. Talballa et al:: AFS Trans., 76-122 (1975), 775.

5) G. A. Irons: Ironmaking Steelmaking, 16 (1989), 28.

6) Y. F. Zhao and G. A. Irons: Proc. of SCANINJECT V, Mefos and Jernkontoret, Lulea, Sweden, (1989), 63.

7) P. E. Plantz: Chap. 6, Modern Methods of Particle Size Analysis, ed. by H. G. Barth, John Wiley \& Sons, New York, (1984).

8) H. N. Frock: Chapt. 10, Particle Size Distribution: Assessment and Characterization, ed. by T. Provder, Amer. Chem. Soc., Washington DC, (1987).

9) P. J. Koros, T. H. Bienosek, J. H. Kaminsky and R. G. Petrushka: SCANINJECT $V$, Mefos and Jernkontoret, Lulea, Sweden, (1989), 183.

10) T. Allen: Particle Size Measurement, Powder Technology Series, 
ISIJ International, Vol. 34 (1994), No. 2

Third Ed. Chapman and Hall, (1981).

11) Y. F. Zhao: Ph. D. Thesis, McMaster Univ., (1992).

12) W. E. Ranz and W. R. Marshall: Chem. Eng. Prog., 48 (1952), 141

13) Y. Yano, N. Yamaguchi and T. Adachi: J. Chem. Eng. Jpn., 7 (1974), 255.

14) D. Mazumdar and R. I. L. Guthrie: Metall Trans. B, 16B (1985), 83.

15) S. Ohguchi and D. G. C. Robertson: Ironmaking Steelmaking, 11
(1984), 262.

16) Handbook of Chemistry and Physics, Vol. 51, ed. by R. J. Weast, (1970), B-77.

17) L. R. Farias and G. A. Irons: Metall. Trans. B, 16B (1985), 211.

18) L. Svarovsky: Powder Testing Guide: Methods of Measuring the Physical Properties of Bulk Powders, Elsevier Applied Sci., (1987).

19) W. K. Kodatsky and A. Gonzales: European Patent 85115081.3, (1985).

20) A. Hacetoglu and W. K. Kodatsky: U. S. Patent 4528031 , (1985). 